\title{
ON POLYNOMIALS IN SELF-ADJOINT OPERATORS IN SPACES WITH AN INDEFINITE METRIC $\left(^{1}\right)$
}

\author{
BY \\ C. Y. LO
}

1. Introduction. Let $H$ be a Hilbert space $\left({ }^{2}\right)$ with the usual inner product $[\cdot, \cdot]$ and norm $\left({ }^{3}\right)$ and with an indefinite inner product $(\cdot, \cdot)$ which, for some orthogonal decomposition $H=H_{1} \oplus H_{2}$ in $H$, is defined by

$$
(x, y)=\left[x_{1}, y_{1}\right]-\left[x_{2}, y_{2}\right] \text { for all } x, y \in H,
$$

where

$$
\begin{array}{ll}
x=x_{1}+x_{2}, & y=y_{1}+y_{2}, \\
x_{1}, y_{1} \in H_{1}, & x_{2}, y_{2} \in H_{2},
\end{array}
$$

and $\operatorname{dim} H_{1}=\kappa$, a positive integer. Such a space $H$ will be called a space $\Pi_{\kappa}$ with an indefinite metric. Another, axiomatic definition of the space $\Pi_{\kappa}$ was given by I. S. Iohvidov and M. G. Krein in [1]; we follow here their terminology, unless otherwise stated, and use the results of their paper.

A linear operator $A$ in $\Pi_{\kappa}$ is called symmetric if it maps a dense domain $D(A)\left(^{4}\right)$ in $\Pi_{\kappa}$ into $\Pi_{\kappa}$ and has the property,

$$
(A x, y)=(x, A y) \text { for all } x, y \in D(A) .
$$

A linear operator $A^{*}$ defined in $\Pi_{x}$ is called the adjoint operator of a linear operator $A$ with a dense domain $D(A)$ in $\Pi_{x}$ if $A^{*}$ is the maximum operator such that

$$
(A x, y)=\left(x, A^{*} y\right) \text { for all } x \in D(A) \text { and all } y \in D\left(A^{*}\right) \text {. }
$$

A symmetric operator is said to be maximal if it has no proper symmetric extension.

A symmetric operator is said to be self-adjoint if $A=A^{*}$.

It is well known in the theory of operators in Hilbert spaces that any two complex conjugate polynomials in a self-adjoint operator are adjoint to each other. We find that the same property holds for polynomials in a self-adjoint operator in the space $\Pi_{\kappa}$ with an indefinite metric. Moreover, if there exists a pair of complex conjugate

Received by the editors August 14, 1966 and, in revised form, October 10, 1966.

(1) This paper is supported by Canadian National Research Council. Part of the results are from the author's Ph.D. thesis.

(2) $H$ is not necessarily separable.

(3) The topology in this paper is the norm topology.

$\left.{ }^{4}\right)$ We shall always denote the domain of an operator $A$ by $D(A)$. 
polynomials in a symmetric operator one of which is adjoint to the other, then this operator is self-adjoint. We shall prove these assertions in this paper.

2. Closed isometric operators. We shall prove here a theorem on isometric operators for later use. Isometric operators in $\Pi_{x}$ are, in general, not continuous. However, a closed isometric operator in $\Pi_{\kappa}$, as we shall show, is continuous.

Definition 2.1. A linear operator $V$ is said to be isometric if

$$
(V x, V x)=(x, x) \text { for all } x \in D(V) \text {. }
$$

Definition 2.2. Let $\Pi_{x}=P \oplus N$, where $P$ is a positive $\kappa$-dimensional subspace and $N$ is the orthogonal complement of $P$. An operator $J$ is called a metric operator if it is defined by the relation,

$$
J(x)=x_{P}-x_{N} \text { for all } x \in \Pi_{\kappa}
$$

where $x=x_{P}+x_{N}, x_{P} \in P$ and $x_{N} \in N$. The new scalar product $[x, y]_{J}=(x, J y)$ is called a $J$-metric and the new norm $|x|_{J}=\left([x, x]_{J}\right)^{1 / 2}$ is called a $J$-norm. By Theorem 1.2 in $[1, \S 2]$ all the $J$-norms are topologically equivalent.

Notation. For any two linear manifolds $L, M$ the notation $L \oplus M$ represents that $(x, y)=0$ for all $x \in L$ and all $y \in M$. The notation $L \oplus_{J} M$ represents that $L \oplus M$ and $[x, y]_{J}=0$ for all $x \in L$ and all $y \in M$ for some metric operator $J$.

THEOREM 2.3. If $V$ is a closed isometric operator, then $V$ is a continuous operator with a closed domain $D(V)$ and a closed range $R(V)$.

Proof. Let $D_{+}(V)$ be a positive subspace of $D(V)$ with the greatest possible dimension and $R_{+}(V)=V D_{+}(V)$. Since $V$ is an isometric operator, the subspace $R_{+}(V)$ is a maximal positive subspace of $R(V)$, having the same dimension as $D_{+}(V)$. We can have the resolutions

$$
D(V)=D_{+}(V) \oplus_{J} D_{-}^{0}(V) \text { and } R(V)=R_{+}(V) \oplus_{J^{\prime}} R_{-}^{0}(V)
$$

where $D_{-}^{0}(V)$ and $R_{-}^{0}(V)$ are nonpositive orthogonal complements of $D_{+}(V)$ and $R_{+}(V)$ in $D(V)$ and $R(V)$ respectively. If the scalar product degenerates on $D_{-}^{0}(V)$, then by Theorem 1.7 in $[1, \S 3]$ we have

$$
D_{-}^{0}(V)=D_{0}(V) \oplus_{J} D_{-}(V),
$$

where $D_{0}(V)$ is the isotropic subspace of the linear manifold $D_{-}^{0}(V)$ and $D_{-}(V)$ is a negative linear manifold. Clearly $R_{0}(V)=V D_{0}(V)$ is the isotropic subspace of the linear manifold $R_{-}^{0}(V)$ and $R_{-}(V)=V D_{-}(V)$ is a negative linear manifold. Obviously we have

$$
R_{-}^{0}(V)=V D_{0}(V) \oplus V D_{-}(V)
$$

Thus we have the resolutions,

$$
D(V)=D_{+}(V) \oplus_{J} D_{0}(V) \oplus_{J} D_{-}(V)
$$

and

$$
R(V)=R_{+}(V) \oplus{ }_{J} R_{0}(V) \oplus R_{-}(V)
$$


If $\mathrm{cl}\left(R_{-}(V)\right)$, the closure of $R_{-}(V)$, is a negative subspace then the theorem is a direct consequence of Theorem 4.3 in $[1, \S 15]$. Thus it remains to prove that cl $\left(R_{-}(V)\right)$ is a negative subspace.

Assuming that the nonpositive subspace $\mathrm{cl}\left(R_{-}(V)\right)$ is a degenerate subspace, by Theorem 1.7 in $[1, \S 3]$ we have the decomposition

$$
\operatorname{cl}\left(R_{-}(V)\right)=N \oplus_{J^{\prime}} R_{-}^{\prime}
$$

where $N$ is the isotropic subspace of $\mathrm{cl}\left(R_{-}(V)\right)$ and $R_{-}^{\prime}$ is a negative subspace. Similarly we have the decomposition

$$
\operatorname{cl}\left(D_{-}(V)\right)=M \oplus_{J} D_{-}^{\prime},
$$

where $M$ is the isotropic subspace of $\mathrm{cl}\left(D_{-}(V)\right)$ and $D_{-}^{\prime}$ is a negative subspace.

Now let $z_{0} \in N$. Then there exists a sequence $\left\{x_{n}\right\}_{0}^{\infty}$ in $D_{-}(V)$ such that $\left\{y_{n}=V x_{n}\right\}_{0}^{\infty}$ is a Cauchy sequence in $R_{-}(V)$, having $z_{0}$ as its limit. From (2.3) and (2.4) we have

$$
x_{n}=x_{0 n}+x_{n}^{\prime}, \quad y_{n}=y_{0 n}+y_{n}^{\prime},
$$

where $x_{0 n} \in M, x_{n}^{\prime} \in D_{-}^{\prime}, y_{0 n} \in N$ and $y_{n}^{\prime} \in R^{\prime}$ for $n=0,1,2, \ldots$ Clearly we have

$$
\left(x_{n}^{\prime}, x_{n}^{\prime}\right)=\left(x_{n}, x_{n}\right)=\left(y_{n}, y_{n}\right)=\left(y_{n}^{\prime}, y_{n}^{\prime}\right)
$$

for $n=0,1,2, \ldots$. Since the scalar product $(.,$.$) is continuous in both arguments$ we have

$$
\lim _{n \rightarrow \infty}\left(y_{n}, y_{n}\right)=\left(z_{0}, z_{0}\right)=0 .
$$

It follows from (2.5) that

$$
\begin{aligned}
\lim _{n \rightarrow \infty}\left[x_{n}^{\prime}, x_{n}^{\prime}\right]_{J} & =-\lim _{n \rightarrow \infty}\left(x_{n}^{\prime}, x_{n}^{\prime}\right)=-\lim _{n \rightarrow \infty}\left(y_{n}^{\prime}, y_{n}^{\prime}\right) \\
& =\lim _{n \rightarrow \infty}\left[y_{n}^{\prime}, y_{n}^{\prime}\right]_{J^{\prime}}=-\lim _{n \rightarrow \infty}\left(y_{n}, y_{n}\right)=0 .
\end{aligned}
$$

In other words, each of the sequences $\left\{x_{n}^{\prime}\right\}_{0}^{\infty}$ and $\left\{y_{n}^{\prime}\right\}_{0}^{\infty}$ converges to the zero vector $\theta$. Hence the sequence $\left\{y_{0 n}\right\}_{0}^{\infty}$ converges to $z_{0}$.

If the sequence $\left\{x_{0 n}\right\}_{0}^{\infty}$ has a Cauchy subsequence with a limit $x \in M$, then $z_{0}=V x$ and $x \in D_{-}(V)$ since $V$ is a closed operator. It follows that $z_{0} \in N \cap R_{-}(V)$, that is $z_{0}=\theta$.

If the sequence $\left\{x_{0 n}\right\}_{0}^{\infty}$ had no Cauchy subsequence, then it would have an unbounded subsequence $\left\{x_{0 k}\right\}_{0}^{\infty}$ such that $\left|x_{0 k}\right|_{J}=d_{k}>k+1$ for $k=0,1,2, \ldots$, since $M$ is a finite dimensional subspace by Lemma 1.2 in $[1, \S 1]$.

We define a sequence

$$
w_{k}=x_{k} / d_{k}=x_{0 k} / d_{k}+x_{k}^{\prime} / d_{k} \text { for } k=0,1,2, \ldots
$$

The sequence $\left\{w_{k}\right\}_{0}^{\infty}$ is clearly in $D_{-}(V)$ and the sequence $\left\{V\left(w_{k}\right)=y_{k} / d_{k}\right\}$ is clearly a Cauchy sequence in $R_{-}(V)$ with the limit $\theta$. The sequence $\left\{x_{k}^{\prime} / d_{k}\right\}_{0}^{\infty}$ converges to $\theta$ and the sequence $\left\{x_{0 k} \mid d_{k}\right\}_{0}^{\infty}$ is bounded in $M$ with $\left.\left|x_{0 k}\right| d_{k}\right|_{J}=1$ for $k=0,1,2, \ldots$ 
Let $\left\{x_{0 m} / d_{m}\right\}_{0}^{\infty}$ be a Cauchy subsequence of $\left\{x_{0 k} / d_{k}\right\}_{0}^{\infty}$, with limit $w_{0} \in M$. It follows that the corresponding subsequence $\left\{w_{m}\right\}_{0}^{\infty}$ is also a Cauchy sequence with limit $w_{0}$. Since $V$ is a closed operator, we have $V\left(w_{0}\right)=\theta$ and $w_{0} \in D_{-}(V)$, that is $w_{0}=\theta$. But $\left|w_{0}\right|_{J}=1$ since $w_{0}$ is the limit of the sequence $\left\{x_{0 m} / d_{m}\right\}_{0}^{\infty}$. This contradiction implies that the sequence $\left\{x_{0 n}\right\}_{0}^{\infty}$ is bounded and hence $z_{0}=\theta$. In other words $N=\{\theta\}$. Now it is easy to show that $D(V)$ and $R(V)$ are closed. The theorem is proven.

3. Polynomials in self-adjoint operators in the space $\Pi_{x}$. Having proven Theorem 2.3 we are now able to investigate some properties of a symmetric operator by using Cayley-von Neumann transformation. Since every symmetric operator has a closed symmetric extension (see $\$ 6$ in [1]), we center our attention on closed symmetric operators.

Let $A$ be a closed symmetric operator with a dense domain $D(A)$. There exists a nonreal number $\zeta$ which is not a proper value of $A$ since a symmetric operator in $\Pi_{x}$ can have at most $2 \kappa$ nonreal proper values (see 1 of $\S 8$ in [1]). We define an operator $V$ by the following formulae:

$$
y=(A x-\zeta x), \quad V y=(A x-\zeta x) \text { for } x \in D(A),
$$

where $\xi$ is the complex conjugate of $\zeta$ or symbolically,

$$
V=(A-\zeta I)(A-\zeta I)^{-1} \text { and } D(V)=(A-\zeta I) D(A) .
$$

The operator $V$ is clearly a closed isometric operator. It follows from Theorem 2.3 that $V$ is a continuous operator with a closed domain $D(V)=(A-\zeta I) D(A)$. Now it is easy to see that the operator $(A-\zeta I)^{-1}$ is continuous. Thus we have proven the following theorem.

THEOREM 3.1. Let $A$ be a closed symmetric operator with a dense domain $D(A)$. If $\zeta$ is a nonreal number which is not a proper value of $A$, then the operator $(A-\zeta I)^{-1}$ is continuous with a closed domain $(A-\zeta I) D(A)$.

Before we prove our main theorem, we need to establish a few lemmas for later use.

LEMMA 3.2. Let $A$ be a linear operator in a linear space $\Pi$ and let $\zeta$ be a complex number. If $(A-\zeta I) D\left(A^{m}\right) \supset D\left(A^{m}\right)$ for some positive integer $m$, then $(A-\zeta I) D\left(A^{n}\right)$ $=D\left(A^{n-1}\right)$ for all natural numbers $n>m$.

Proof. We shall prove this lemma by induction. Let $n=m+1$. It is obvious that $(A-\zeta I) D\left(A^{m+1}\right) \subset D\left(A^{m}\right)$. We need to prove only the reverse inclusion. For any $x \in D\left(A^{m}\right)$ by assumption there exists $y \in D\left(A^{m}\right)$ such that $(A-\zeta I) y=x$. It follows that $A y \in D\left(A^{m}\right)$, that is $y \in D\left(A^{m+1}\right)$. Hence $(A-\zeta I) D\left(A^{m+1}\right) \supset D\left(A^{m}\right)$ and we have proved our lemma for $n=m+1$. Using the same kind of arguments we can prove the lemma for the case $n=k+1$ by assuming it is true for $n=k$. The lemma is proven. 
LEMMA 3.3. Let $A$ be a self-adjoint operator in $\Pi_{x}$ and let $P(\lambda)=\prod_{i=1}^{n}\left(\lambda-\zeta_{i}\right)$ be a polynomial with nonreal roots. If no root of $P(\lambda)$ is a proper value of $A$, then $\left(^{5}\right)$ $P(A) D\left(A^{m}\right)=D\left(A^{m-n}\right)$ for $m>n$, where $m, n$ are natural numbers.

Proof. By Theorem 2.9 in $[1, \S 9]$ we have $\left(A-\zeta_{i} I\right) D(A)=\Pi_{\kappa}$ for $i=1,2, \ldots, n$. Thus this lemma follows Lemma 3.2 immediately.

LEMMA 3.4. Let $A$ be a maximal symmetric operator in $\Pi_{\kappa}$. Then $D\left(A^{n}\right)$ is dense in $\Pi_{x}$ for any natural number $n$.

Proof. We shall prove this lemma by induction. For $n=1$ the lemma is true by the definition of a maximal symmetric operator.

Now we assume this lemma is true for $n=m$. By Theorem 2.9 in $[1, \S 9]$ we have a pair of complex numbers $(\zeta, \zeta)$ such that

$$
(A-\zeta) D(A)=\Pi_{\kappa} \text { and }(A-\bar{\zeta}) D(A)=M,
$$

where $M$ is a nondegenerate subspace, containing a $\kappa$-dimensional positive subspace. Thus by Theorem 1.5 in $[1, \S 3]$ we have the resolution

$$
\Pi_{\kappa}=M \oplus_{J} N,
$$

where $N$ is the orthogonal complement of $M$. By Lemma 3.2 we have

$$
(A-\zeta) D\left(A^{m+1}\right)=D\left(A^{m}\right)
$$

from relation (3.1).

Now for any $x \in \Pi_{x}$ we have $y \in D(A)$ such that $x=(A-\zeta) y=\left(A^{*}-\zeta\right) y$ by relation (3.1). From relation (3.2) we have $y=y_{M}+y_{N}$, when $y_{M} \in M$ and $y_{N} \in N$. It follows from (3.1) there exists $z \in D(A)$ such that $y_{M}=(A-\bar{\zeta}) z$. Since $\left(A^{*}-\zeta\right) y_{N}$ $=\theta$, we have

$$
x=\left(A^{*}-\zeta(A-\zeta) z\right.
$$

for some $z \in D(A)$. If $x \in \Pi_{x}$ and $\left(x, D\left(A^{m+1}\right)\right)=0$ then

$$
\begin{aligned}
0 & =\left(x, D\left(A^{m+1}\right)\right)=\left(\left(A^{*}-\zeta\right)(A-\zeta) z, D\left(A^{m+1}\right)\right) \\
& =\left((A-\zeta) z,(A-\zeta) D\left(A^{m+1}\right)\right) \\
& =\left((A-\zeta) z,(A-\zeta) D\left(A^{m+1}\right)\right) .
\end{aligned}
$$

It follows from (3.3) that $0=\left((A-\zeta) z, D\left(A^{m}\right)\right)$. Hence we have $(A-\zeta) z=\theta$ by assumption. Since $\zeta$ is not a proper value of $A$ we must have $z=\theta$. Thus from (3.4) we conclude that $x=\theta$. It thus follows that $D\left(A^{m+1}\right)$ is dense in $\Pi_{x}$. The lemma is proved.

LEMMA 3.5. Let $P(\lambda)$ be a polynomial of degree $n$ and let $F$ be a finite set of $m$ complex numbers. Then we can always find a nonreal number $\zeta_{0}$ such that all the roots of the polynomial $P(\lambda)-\zeta_{0}$ are nonreal and these roots are not in the set $F$.

(5) We agree that for any operator $A, A^{0}=I$ where $I$ is the identity operator. 
Proof. It is easy to see that if $\zeta$ and $\zeta^{\prime}$ are different numbers, then the polynomials $P(\lambda)-\zeta$ and $P(\lambda)-\zeta^{\prime}$ have no common factors. Hence for only a finite number of complex numbers $\zeta_{i}, i=1,2, \ldots, m^{\prime}\left(m^{\prime} \leqq m\right)$ does the corresponding polynomial $P(\lambda)-\zeta_{i}$ have roots in the set $F$. It thus follows that for any complex number $\zeta$ such that $\operatorname{Re} \zeta>\operatorname{Re} \zeta_{i}, i=1,2, \ldots, m^{\prime}$ the polynomial $P(\lambda)-\zeta$ has no roots in $F$.

Let $P(\lambda)=P^{(1)}(\lambda)+i P^{(2)}(\lambda)$, where $P^{(1)}(\lambda)$ and $P^{(2)}(\lambda)$ are real polynomials of degree at most $n$. Let $\zeta=c+i d$, where $c \neq 0$ and $d$ are real numbers such that $c>\operatorname{Re}\left(\zeta_{i}\right), i=1,2, \ldots, m^{\prime}$. If $\lambda_{0}$ is a real root of the polynomial $P(\lambda)-\zeta$, then we have

$$
P^{(1)}\left(\lambda_{0}\right)-c=0
$$

and

$$
P^{(2)}\left(\lambda_{0}\right)-d=0
$$

It is clear that for a fixed real number $c$, there exist at most $n \lambda_{0}$ 's satisfying the relation (3.5). It thus follows that we can find a real number $d_{0} \neq 0$ such that the polynomial $P(\lambda)-\left(c+i d_{0}\right)$ has no real roots. Hence the number $\zeta_{0}=c+i d_{0}$ is the desired nonreal number. The lemma is proved.

LEMMA 3.6. If $A$ is a closed linear operator in $\Pi_{\kappa}$ then the adjoint of the adjoint of $A$ is $A$.

Proof. Let $J$ be a metric operator. Clearly $J A$ is also a closed linear operator since $J$ is a bicontinuous linear operator by Theorem 1.2 in [1, §2]. Let us denote the adjoint of $J A$ with respect to the $J$-metric by $(J A)^{J}$. Since the space $\Pi_{\kappa}$ together with a $J$-metric is a Hilbert space, we have $(J A)^{J J}=J A$. It is obvious that for any linear operator $B$ with a dense domain $(J B)^{J}=J B^{*}$. It thus follows that $J A=\left(J A^{*}\right)^{J}$ $=J A^{* *}$. Since $J$ is bijective, we have $A=A^{* *}$. The lemma is proved.

THEOREM 3.7. Let $A$ be a symmetric operator in $\Pi_{\kappa}$ and let $P(\lambda)$ and $\bar{P}(\lambda)$ be complex conjugate polynomials of degree $n$. Then the operator $\bar{P}(A)$ is adjoint to $P(A)$ if and only if $A$ is a self-adjoint operator.

Proof. (1). Let $A$ be a self-adjoint operator. Since $A$ can have only a finite number of nonreal proper values, it follows that by Lemma 3.5 we can find a nonreal number $\zeta$ such that the polynomial $P(\lambda)-\zeta$ has no root which is a proper value of $A$ or its complex conjugate. Hence $\bar{P}(\lambda)-\zeta$ also has no root which is a proper value of $A$. It follows that $\zeta$ and $\zeta$ are not proper values of $P(A)$ and $\bar{P}(A)$ respectively.

It is clear that $D(P(A))=D\left(A^{n}\right)=\bar{D}(P(A))$. Since $D\left(A^{n}\right)$ is dense in $\Pi_{\kappa}$ by Lemma 3.4 , the adjoint operator $P(A)^{*}$ of $P(A)$ exists. Obviously we have $P(A)^{*} \supset P(A)$. Therefore it is sufficient to prove $D\left(A^{n}\right) \supset D\left(P(A)^{*}\right)$ in order to prove $\bar{P}(A)=P(A)^{*}$. 
By Lemma 3.3 we have

$$
(P(A)-\zeta I) D\left(A^{n}\right)=\Pi_{x}=(\bar{P}(A)-\xi I) D\left(A^{n}\right) .
$$

For any $x \in D\left(P(A)^{*}\right)$ there exists $z \in D\left(A^{n}\right)$ such that

$$
\left(P(A)^{*}-\xi I\right) x=(\bar{P}(A)-\xi I) z
$$

by relation (3.7). In other words, we have $\left(P(A)^{*}-\xi I\right)(x-z)=\theta$. It thus follows that for all $y \in D\left(A^{n}\right)$ we have

$$
0=\left(\left(P(A)^{*}-\xi I\right)(x-z), y\right)=((x-z),(P(A)-\zeta I) y) .
$$

Since $(P(A)-\zeta I) D\left(A^{n}\right)=\Pi_{x}$, we have $x-z=\theta$, that is $x=z \in D\left(A^{n}\right)$. Similarly we can prove $\bar{P}(A)^{*}=P(A)$. The first part of the theorem is proved.

(2) Now let $P(A)$ and $\bar{P}(A)$ be adjoint to each other. We choose $\zeta$ such that the polynomial $P(\lambda)-\zeta=\prod_{i=1}^{n}\left(\lambda-\zeta_{i}\right)$ has no root which is a proper value or its complex conjugate of the operator $\bar{A}$, the closed extension of $A$. It thus follows $\zeta$ and $\zeta$ are not proper values of $P(A)$ and $\bar{P}(A)$ respectively.

We shall show that $(P(A)-\zeta I) D\left(A^{n}\right)$ is dense in $\Pi_{x}$. Let $x \in \Pi_{x}$ be such that $(x,(P(A)-\zeta I) y)=0$ for all $y \in D\left(A^{n}\right)$. It follows that for all $y \in D\left(A^{n}\right)$ we have

$$
0=\left(\left(P(A)^{*}-\xi I\right) x, y\right)=((\bar{P}(A)-\xi I) x, y) .
$$

Since $D\left(A^{n}\right)$ is dense in $\Pi_{x}$ by Lemma 3.4, we have $(\bar{P}(A)-\xi I) x=\theta$, the zero vector. As $\xi$ is not a proper value of $\bar{P}(A), x$ must be the zero vector $\theta$. Therefore $(P(A)-\zeta I) D\left(A^{n}\right)$ is dense in $\Pi_{\kappa}$.

We shall show $(P(A)-\zeta I) D\left(A^{n}\right)=\Pi_{\kappa}$. Let us define an operator $U$ in the $\Pi_{\kappa}$ by the formulae:

$$
y=(P(A)-\zeta I) x, \quad U y=(\bar{P}(A)-\zeta I) x
$$

for all $x \in D\left(A^{n}\right)$. Clearly $U$ is an isometric operator with dense domain in $\Pi_{x}$. The operator $U$ is bicontinuous by Theorem 4.3 in $[1, \S 15]$. Since the operators $\left(A-\zeta_{i}\right)^{-1}$, $i=1,2, \ldots, n$ are continuous by Theorem 3.1 , the operator $(P(A)-\zeta I)^{-1}=$ $\prod_{i=1}^{n}\left(A-\zeta_{i}\right)^{-1}$ is also continuous. As $\bar{P}(A)=P(A)^{*}$ is a closed operator, it follows that $U$ and $P(A)-\zeta I$ are also closed operators. Applying Theorem 2.3 we conclude that $(P(A)-\zeta I) D\left(A^{n}\right)=D(U)$ is a subspace. Since it is dense in $\Pi_{\kappa}$, it can only be the whole space $\Pi_{x}$. As $P(A)-\zeta I$ is a closed operator, the operator $P(A)$ must be a closed operator. It thus follows from Lemma 3.6 that $P(A)=P(A)^{* *}$. Hence by similar arguments we have $(\bar{P}(A)-\xi I) D\left(A^{n}\right)=\Pi_{x}$.

We shall show that $\left(A-\zeta_{1}\right) D(A)=\Pi_{x}=\left(A-\zeta_{1}\right) D(A)$. It is sufficient to prove $\prod_{i=2}^{n}\left(A-\zeta_{i}\right) D\left(A^{n}\right) \supset D(A)$. Since $\prod_{i=1}^{n}\left(A-\zeta_{i}\right) D\left(A^{n}\right)=\Pi_{x}$, for any $x \in D(A)$ there exists $x^{\prime} \in \prod_{i=2}^{n}\left(A-\zeta_{i}\right) D\left(A^{n}\right)$ such that $\left(A-\zeta_{i}\right) x^{\prime}=\left(A-\zeta_{1}\right) x$, that is $\left(A-\zeta_{1}\right)\left(x^{\prime}-x\right)$ $=\theta$. Since $\zeta_{1}$ is not a proper value of $A$, we must have $x=x^{\prime}$.

We shall show that $A=A^{*}$. Since $D(A) \supset D\left(A^{n}\right)$ and $\operatorname{cl}\left(D\left(A^{n}\right)\right)=\Pi_{x}, A^{*}$ exists. It is obvious $A^{*} \supset A$; therefore it is sufficient to prove $D\left(A^{*}\right) \subset D(A)$. Since 
$\left(A-\zeta_{1}\right) D(A)=\Pi_{x}$, for any $x \in D\left(A^{*}\right)$ there exists $y \in D(A)$ such that $\left(A^{*}-\zeta_{1}\right) x$ $=\left(A-\zeta_{1}\right) y$, that is $\left(A^{*}-\zeta_{1}\right)(x-y)=\theta$. It follows that $\left(\left(A^{*}-\zeta_{1}\right)(x-y), z\right)=0$ for all $z \in D(A)$. Hence $\left((x-y),\left(A-\xi_{1}\right) z\right)=0$ for all $z \in D(A)$. As $(A-\zeta) D(A)=\Pi_{\kappa}$, we have $x-y=\theta$, that is $x=y$. So we have $D\left(A^{*}\right)=D(A)$ and $A=A^{*}$. The theorem is completely proven.

THEOREM 3.8. Let $A$ be a symmetric operator in $\Pi_{x}$ and let $P(\lambda)$ be a real polynomial of degree greater than one. Then $P(A)$ is a maximal symmetric operator if and only if $A$ is self-adjoint.

Proof. If $A$ is self-adjoint, the operator $P(A)$ must be self-adjoint, that is maximal, by Theorem 3.7. Now let $P(A)$ be maximal and let $\tilde{A}$ be a maximal symmetric extension of the operator $A$. Then $P(A)=P(\tilde{A})$ must hold. If $\tilde{A}$ is self-adjoint, then $P(A)$ is self-adjoint and consequently $A$ is self-adjoint by Theorem 3.7. If $\tilde{A}$ is not self-adjoint, we shall show $P(A)=P(\tilde{A})$ can not be maximal. If $P(\tilde{A})$ were maximal there exists a nonreal number $\zeta$ which is not a proper value of $P(\widetilde{A})$ such that

$$
(P(\tilde{A})-\zeta) D\left(\tilde{A}^{n}\right)=\Pi_{\kappa}=\prod_{i=1}^{n}\left(\tilde{A}-\zeta_{i}\right) D\left(\tilde{A}^{n}\right) .
$$

It follows that the roots of $P(\lambda)-\zeta$ are not proper values of $A$. Since $P(\lambda)$ is a real polynomial of degree at least two and since $\zeta$ is a nonreal number, there exists at least one root of the polynomial $P(\lambda)-\zeta$ in both the upper and the lower half of the complex plane. It thus follows that there exists a root $\zeta_{10}$ such that $\left(\tilde{A}-\zeta_{10}\right) D(A)$ $\neq \Pi_{\kappa}$. Hence we have

$$
\prod_{i=\frac{1}{1}}^{n}\left(\tilde{A}-\zeta_{i}\right) D\left(\tilde{A}^{n}\right) \subset\left(\tilde{A}-\zeta_{i 0}\right) D(\tilde{A}) \neq \Pi_{x} .
$$

This contradiction implies that $A$ must be a self-adjoint operator. The theorem is proven.

ACKNOWLEDGMENTS. This work was done under the supervision and guidance of Professor I. Halperin, Queen's University. It is a pleasure to express my gratitude to Professor I. Halperin for suggesting the problem to me and for his encouragement and guidance.

\section{REFERENCE}

1. I. S. Iohvidov and M. G. Krein, Spectral theory of operators in spaces with an indefinite metric. I, Amer. Math. Soc. Transl. (2) 13 (1960), 105-175; II, Amer. Math. Soc. Transl. (2) 34 (1963), 283-373.

QUEEN'S UNIVERSTTY, KIngston, CANADA 